\title{
Comparison Between the Production of Hibiscus in Kordofan States Using Matlab
}

\author{
Abdel Radi Abdel Rahman Abdel Gadi ${ }^{1}$, Ragaa Mohammed Haj Ebrahim ${ }^{2}$, \\ Nedal Hassan Elbadowi Eljaneid ${ }^{3, *}$ \\ ${ }^{1}$ Department of Mathematics, Faculty of Education, Omdurman Islamic University, Omdurman, Sudan \\ ${ }^{2}$ Department of Mathematics, Faculty of Education, Alzaiem Al Azhary University, Khartoum, Sudan \\ ${ }^{3}$ Department of Mathematics, College of Science, Tabuk University, Tabuk, Saudi Arabia
}

Email address:

needhal@hotmail.com (N. H. E. Eljaneid)

${ }^{*}$ Corresponding author

\section{To cite this article:}

Abdel Radi Abdel Rahman Abdel Gadi, Ragaa Mohammed Haj Ebrahim, Nedal Hassan Elbadowi Eljaneid. Comparison Between the Production of Hibiscus in Kordofan States Using Matlab. Pure and Applied Mathematics Journal. Vol. 8, No. 1, 2019, pp. 10-17. doi: 10.11648/j.pamj.20190801.12

Received: January 23, 2019; Accepted: March 4, 2019; Published: April 18, 2019

\begin{abstract}
Hibiscus (Karkadi) contains plant acids such as malic, chemical and that indicates its high importance for humans. Many industries depend on Karkadi in most of the areas in Sudan due to its economic and medical benefits . In this study the statistical data of Hibiscus will be analyzed using Matlab. This study aims to compare and analyze the data of Hibiscus production in North Korodfan state, west Korodfan state and south Korodfan state in period 2006 to 2016 and if planted areas and harvested areas affect production or not. The applying mathematical method was followed using Matlab and found it is more accurate because the data is converges with the analysis, and arrived to relation between production, planted areas and harvested areas. All this data passed through code that designed by Matlab program for analysis and counting data in the analysis obtained bar graph for all data for each state. And counting production for select state of hibiscus production (high, low and non-exist). A new algorithm is designed based on the type of data and compared results with each year in one state, Matlab functions results showed excellent efficiency with $95 \%$ accuracy for analysis, counting and classification.
\end{abstract}

Keywords: Production, Hibiscus, Matlab

\section{Introduction}

Roselle (Hibiscus Sabdariffa $L$ ) belongs to the family malvacease locally called Karkadi in Sudan is an important annual crop grown successfully in tropical and sub-tropical climate Karkadi is one of the crops cultivated by traditional farmers in western Sudan especially in various parts of Kordofan and Darfour States. The commercial part of the plant is the fleshy calyx sepals surrounding the fruit. When fully developed the fleshy calyx is peeled off from the fruit and dried in the shade to give the dry Karkadi which is the commercial produce of the planed the color of the calyx plays an important role in determining the quality of Karkadi. [17].

Hibiscus is a flowering plant with more than 300 species.
The plant is native in the warm climatic conditions of tropical and subtropical regions. Most varieties are used in a diverse number of ways: from herbal tea to juices, jellies, jams, ice cream, flavors, and the fabrication of paper. The product has different names in different places, like sorrel, roselle cabitutu, vinuela, oseille de guinée, bissap, or karkadeh. [7]

The crimson red color is the characteristic and the most popular desirable color of Karkadi. While other shade and various of color exist, including the white color. [17]

\section{Medicinal and Industrial Applications}

Many medicinal applications of the Roselle plant have been developed around the world. In China it is used to treat hypertension, pyrexia and liver damage, and in Ayurvedic 
medicine. [1]

Recently the sepal extract has been used as an effective treatment against leukemia due to its high content in polyphones, particularly protocatechuic acid. [1]

\section{Ecology and Distribution}

Roselle is relatively a hardy crop; a tropical or subtropical plant requires a monthly rainfall ranging from $130-250 \mathrm{~mm}$ in the first three to four months of growth. Most evidence points to west Africa as the home origin of Kardadi and from there it had spread to their parts of Africa countries including Sudan. It is a short day plant, flowering best with 11 to 12 hour days.

A temperature range between 20 and $35 \mathrm{c}$. without prolonged dull weather available moisture equivalent to $25 \mathrm{~mm}$ month through the growing period together with humid air conditions are most conclusive to optimum growth.

The crop can be grown in wide range of soil type the best being heavy retentive friable loam laterite and poor sandy soil and heavy days are unsatisfactory it does not withstand flooding in view of its deep root system it requires adequate depth.

\section{Areas and Production}

In Kordofan states most of the Karkadi production comes from Um Rowaba and El Rahad localities in North Kordofan State. El Rahadarea is the most popular area for Karkadi production small- scattered area is planted in other parts of Kordofan and Darfur states. Karkadi is grown by traditional farmers in small acreages ranging from under 0.25 to 2 ha few growers, merchants can have up to 20 ha. One factor limiting the size of individual area planted is the difficulty and longtime needed for peeling off the calyx at harvest using hard Workers. Karkadi is usually intercropped or planted in mixtures with other field cropsa like sorghum and Sesame. Afer farmers plant it in pure stand or in borders between farms. The total area planted with Karkadi in Kordofan is variable and highly affected by the prices and status of marketing in the previous season. However, the Karkadi area planted in North Kordofan State amount over $90 \%$ of the total area grown under the crop in the country. [17]

Table 1. Data of North Kordofan State [18].

\begin{tabular}{llll}
\hline Years & Planted area & Harvested area & Production \\
\hline 2006 & 355 & 301 & 14 \\
2007 & 850 & 595 & 21 \\
2008 & 490 & 430 & 16 \\
2009 & 850 & 595 & 21 \\
2010 & 687 & 518 & 18 \\
2011 & 522 & 457 & 30 \\
2012 & 437 & 262 & 18 \\
2013 & 241 & 195 & 14 \\
2014 & 240 & 120 & 8 \\
2015 & 244 & 193 & 14 \\
2016 & 300 & 195 & 9 \\
\hline
\end{tabular}

Table 2. Data of West Kordofan State [18].

\begin{tabular}{llll}
\hline Years & Planted area & Harvested area & Production \\
\hline 2006 & 0 & 0 & 0 \\
2007 & 0 & 0 & 0 \\
2008 & 0 & 0 & 0 \\
2009 & 0 & 0 & 0 \\
2010 & 0 & 0 & 0 \\
2011 & 0 & 0 & 0 \\
2012 & 0 & 0 & 0 \\
2013 & 0 & 0 & 0 \\
2014 & 302 & 227 & 17 \\
2015 & 305 & 238 & 19 \\
2016 & 350 & 298 & 15 \\
\hline
\end{tabular}

Table 3. Data of South Kordofan State [18].

\begin{tabular}{llll}
\hline Years & Planted area & Harvested area & Production \\
\hline 2006 & 16 & 12 & 1 \\
2007 & 30 & 28 & 2 \\
2008 & 32 & 28 & 1 \\
2009 & 30 & 28 & 2 \\
2010 & 50 & 38 & 3 \\
2011 & 70 & 67 & 5 \\
2012 & 50 & 38 & 3 \\
2013 & 70 & 65 & 4 \\
2014 & 37 & 28 & 2 \\
2015 & 43 & 37 & 3 \\
2016 & 10 & 9 & 0 \\
\hline
\end{tabular}

\section{Introduction to Matlab}

Matlab is a program that allows you to carry out computations in a straight forward manner, removing much of the tedium involved in programming. It is extremely useful for creating simulations of neural networks, as well as for general types of data analysis and visualization. [19]

\subsection{Accessing Matlab}

On Unix systems you can enter Matlab with the system command Matlab and exit Matlab with the Matlab command quit or exit. In Microsoft Windows and the Macintosh, just double-click on the Matlab icon:

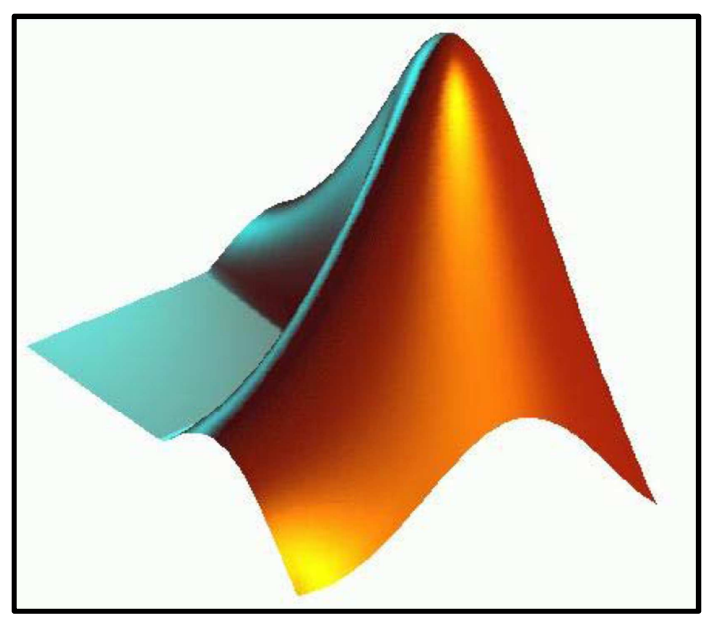

Figure 1. The Matlab Icon [10]. 


\subsection{The Matlab System}

\subsubsection{Development Environment}

This is the set of tools and facilities that help you use Matlab functions and files. Many of these tools are graphical user interfaces. It includes the Matlab desktop and Command Window, a command history.[15]

\subsubsection{The Matlab Desktop}

Matlab has an extensive graphical user interface. When Matlab starts, the main Matlab window appears, containing several windows and menu bars. Not all windows appear in the default configuration. The Desktop menu controls the layout and appearance of the windows and gives you a list of the windows you can use. [12]

\subsubsection{Command Window}

Statup When you cilck the Matlab icon, the Ms windows opens up the standard Matlab -window for you which has the following form:

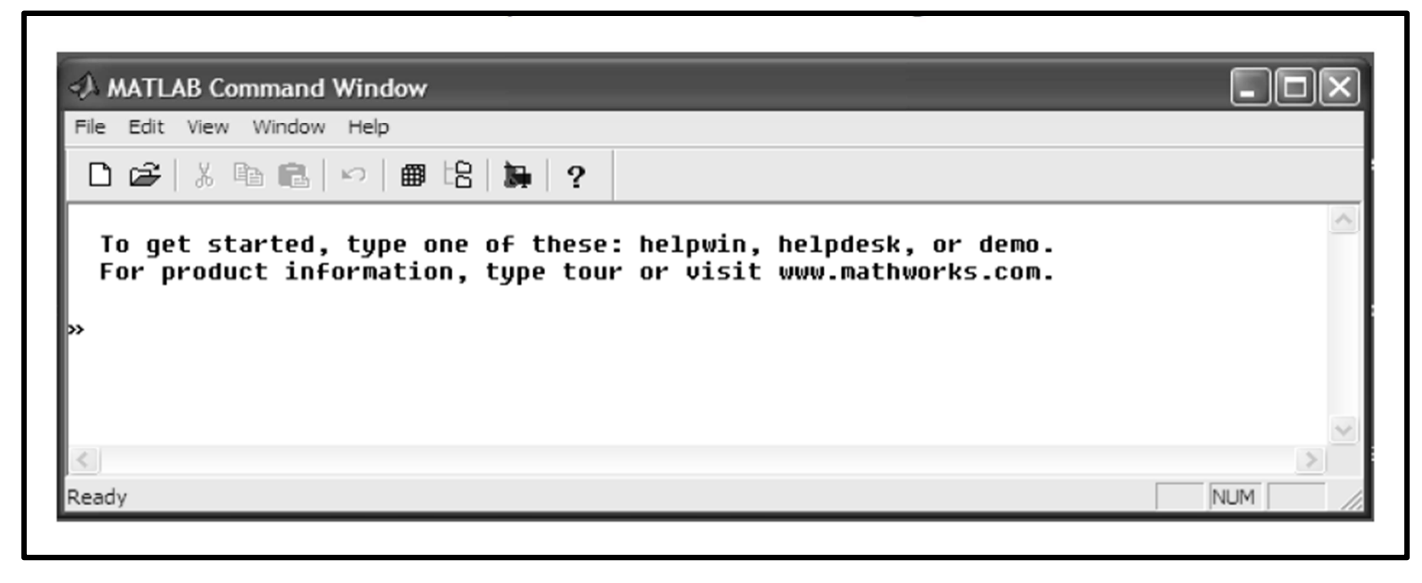

Figure 2. Matlab Command Window.

The white area in the middle is the work area in which the user types - in the commands which are interpreted directly over there and the results are displayed on screen.

The ' $>>$ ' isMatlab prompt indicating that user can typein command here. A previously entered command can be reached with the help of up - arrow and down -arrow buttons on the keyboard. [8]

\subsubsection{Matlab Functions}

Matlab has a wide assortment of built-in functions. You have already seen some of them, such as zeros, rand, and inv. This Table flow describes the more common matrix manipulation functions. For a more complete list.[11]

\subsubsection{Handle Graphics}

Matlab's high-level plotting function was used (plot, surf, etc.). High-level plotting functions produce simple graphs and automate the many mundane decisions you might make in producing a plot, such as the position of the plot, the colour of the axes, the font size, the line thickness, and so on. Matlab's system of Handle Graphic sallows you to control a great many of these "mundane" aspects of plotting, to produce plots that are optimised for communicating the data at hand. The idea behind Handle Graphics is that every object in the figure window (axes, lines, text, surfaces, etc.) has a set of properties.

These properties can be examined using the get command and set to new values using the set command. Every object in the figure window also has a unique identifier (a number) called a handle. The object's handle tells get and set what object you are interested in.[2]

\subsubsection{Two - Dimensional Plots (Bar Graphs)}

A bar graph can quickly be created with the bar command. The bar function can be used to plot bars with heights specified by the variable argument, bar_height_vector, versus the index number of that variable by using bar (bar_height_vector); If instead of the index to the variable, you want to plot bars versus another variable, you can use $\operatorname{bar}(x, y)$, where $x$ and $y$ are equal length vectors, and vector $x$ contains values which are both in ascending order and evenly spaced.

If $x$ is not evenly spaced or in ascending order, the routine will do the best it can do, but the results will most likely not be what you wanted.

If, for example, you want to create a bar graph of the percentage of widgets that passed quality tests versus the assembly line number, you can type assembly line_number $=$

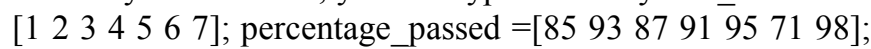
bar (assembly_line_number, percentage_passed);

$x$ label('Assembly Line Number').

$y$ label('Percentage Passed'). [4]

Which will produce the plot shown in Figure 3.

When we plot bar graphs, we may wish to have labels other than the numeric ones that automatically appear on your $x$-axis. In these cases, the simplest way to plot our bar graph is with the bar (bar_height_vector) format.[13] 


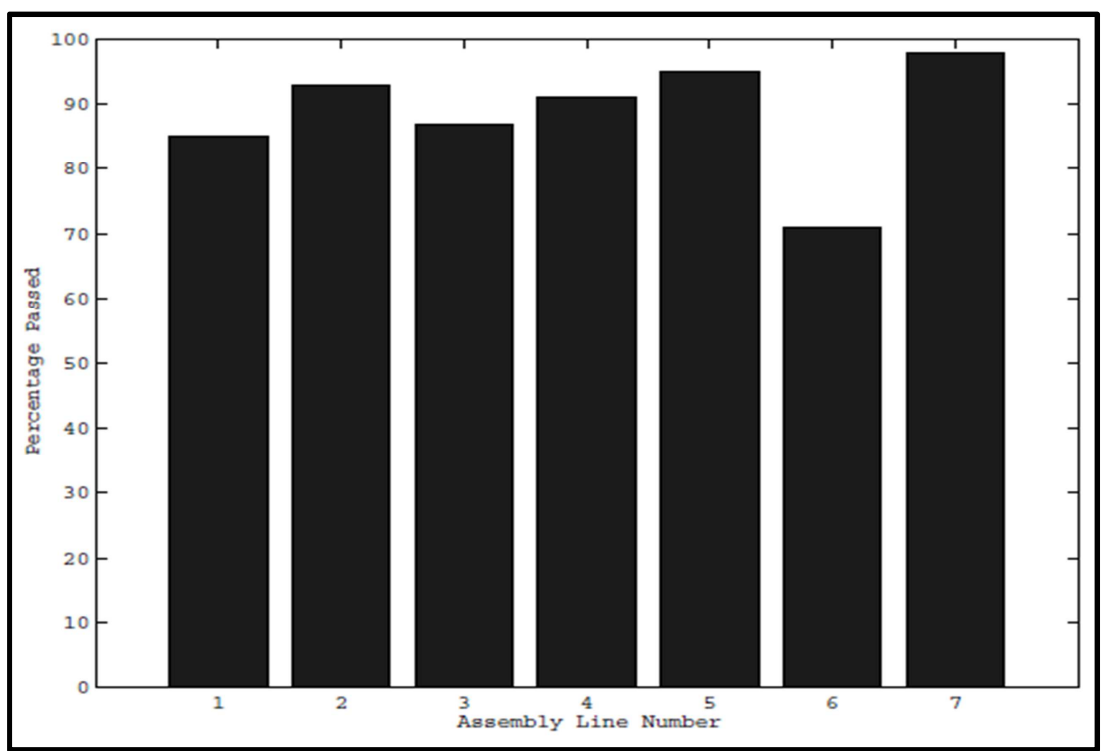

Figure 3. Using the bar Function [13].

\subsubsection{Surfaces in Three-Dimensional Space}

There are two basic commands for plotting surfaces in 3space: mesh and surf. The former produces a transparent "mesh" surface; the latter produces an opaque shaded one. There are two different ways of using each command, one for plotting surfaces in which the $z$ coordinate is given as a function of $x$ and $y$, and one for parametric surfaces in which $x, y$, and $z$ are all given as functions of two other parameters. Let us illustrate the former with mesh and the latter with surf.

To plot $z=f(x, y)$, one begins with ameshgrid command as in the case of contour. For example, the "saddle surface" $z=x_{2}-y_{2}$ can be plotted with

$>[x, y]=$ meshgrid( $(-2: .1: 2,-2: .1: 2)$;

$>Z=x \cdot{ }^{\wedge} 2-y \cdot{ }^{\wedge} 2$;

$>>\operatorname{mesh}(x, y, z)$

The result is shown in Figure 4, although it looks much better on the screen since Matlab shades the surface with a color scheme depending on the $z$ coordinate. We could have gotten an opaque surface instead by replacing mesh with surf. [5]

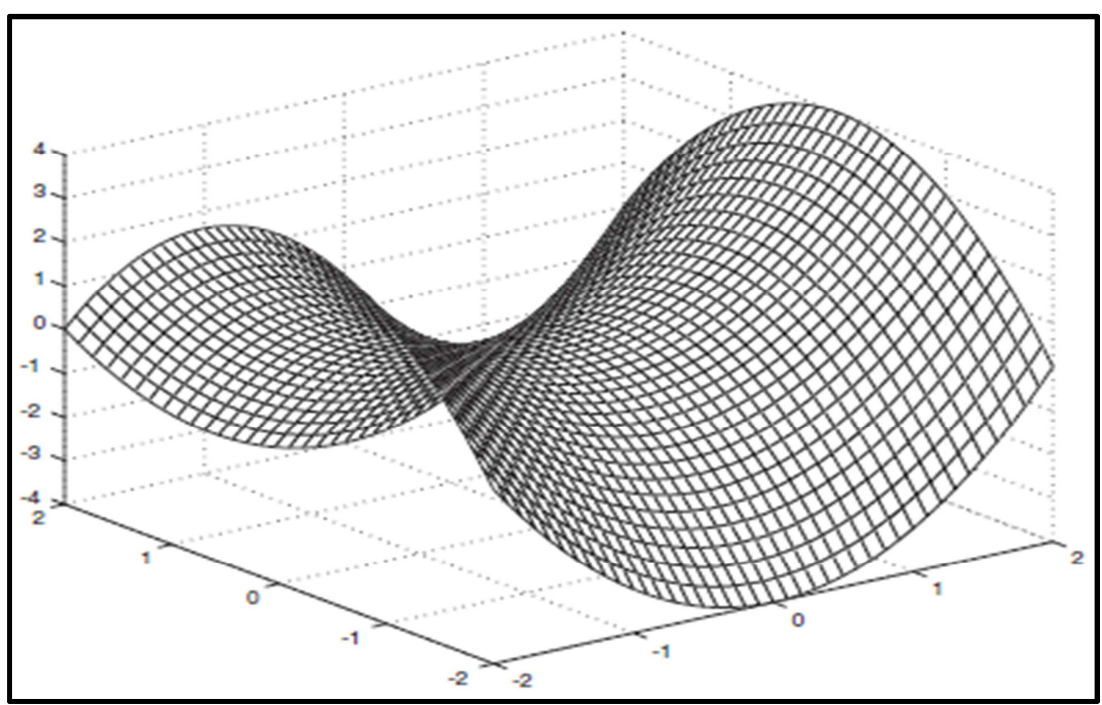

Figure 4. Surfaces in Three-Dimensional Space [3].

\subsubsection{Vectors}

A vector is a one-dimensional array of numbers. Matlab allows you to create column vectors or row vectors. A column vector can be created in Matlab by enclosing a set of semicolon delimited numbers in square brackets. Vectors can have any number of elements. For example, to create a column vector with three elements we write:[6]

$$
\begin{aligned}
& >>z=[2,4,6,8] \\
& z=2468 \\
& >>y=[4-35-281] \\
& y=4-35-281
\end{aligned}
$$


Suppose you want to create a vector of values running from 1 to 9 . Here's how to do it without typing each number:

$>x=1: 9$

$$
x=123456789 .[3]
$$

\section{Main Characteristics of Matlab}

\subsection{The for Loop}

The for statement, or the for loop, is used when it is necessary to repeat statement(s) in a script or function, and when it is known ahead of time how many times the statements will be repeated. The statements that are repeated are called the action of the loop. For example, it may be known that the action of the loop will be repeated five times. The terminology used is that we iterate through the action of the loop five times.

The variable that is used to iterat.[14]

The basic syntax for a loop is:

for $\mathrm{i}=$ array

Commands

end [9]

\subsection{If-else Statements}

If-else if statement may be used to test various conditions before executinga set of statements.

The general form of the if-else if statement isif logical expression 1

statement group 1

else if logical expression 2

statement group 2

else if logical expression 3

statement group 3

else if logical expression 4 statement group 4

end-

A statement group is executed provided the logical expression above it is true.

For example, if logical expression 1 is true, then statement group 1 is executed.[16]

\section{Analysis of Hibiscus Production Data Using Matlab}

Matlab Program used to analyze Hibiscus production data from 2006 up to 2016, a function was established for each state and implement the data of planted area, harvested area, and the annual production in par paragraph form. In par paragraph where $x$ axis refers to the years from 2006 up to 2016, While $y$ axis symbolize the planted and harvested area and production unit. Thus the blue rectangle indicates the planted area, where as the green rectangle refers to the harvested area and the Brown rectangle show as the production.

\subsection{Data Analysis of North Kordufan State}

In North Kordufan State the production is shown in Figure 5 as the highest planted area was in the 2007-2009 although production was not higher however the highest production is achieved in the year 2011 .

Reasons: The scarcity of rain, which ranges between 250$350 \mathrm{~mm}$, distribution of rain , the soil is sandy and tradition agriculture in which Karkadi is grown with another crop sharing the same hole, or interplanting in which Karkadi, is grown in interplanting rows with other crops such as sorghum and millet with Karkadi occupying one row.

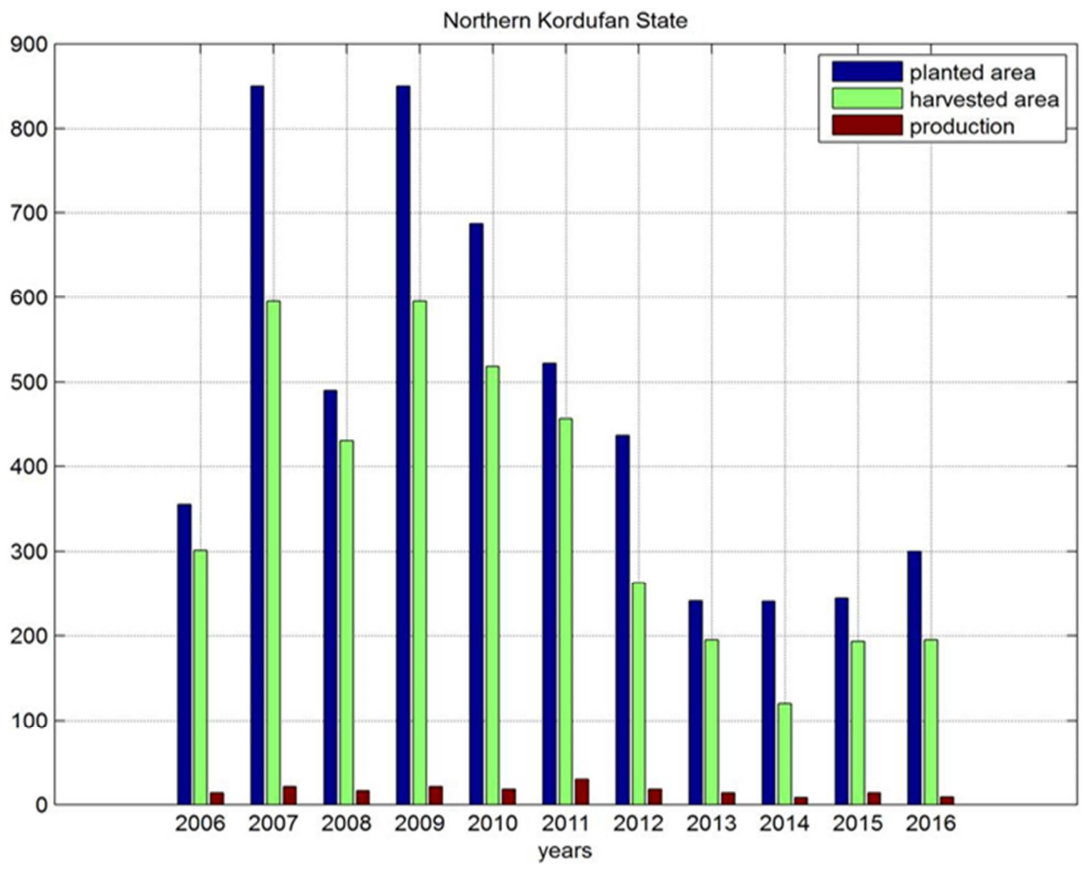

Figure 5. Data Analysis of North Kordufan State. 


\subsection{Data Analysis of West Kordufan State}

The Figure 6 found in west Kordufan State planted and harvesting had been executed is last three years only, and of ten yeas which were under Reasons: Lack of rehabilitation of the irrigation system in the agricultural projects as they are located different states - the northern area lies in northern Kordufan State and the southern area lies in Southern Kordufan State.

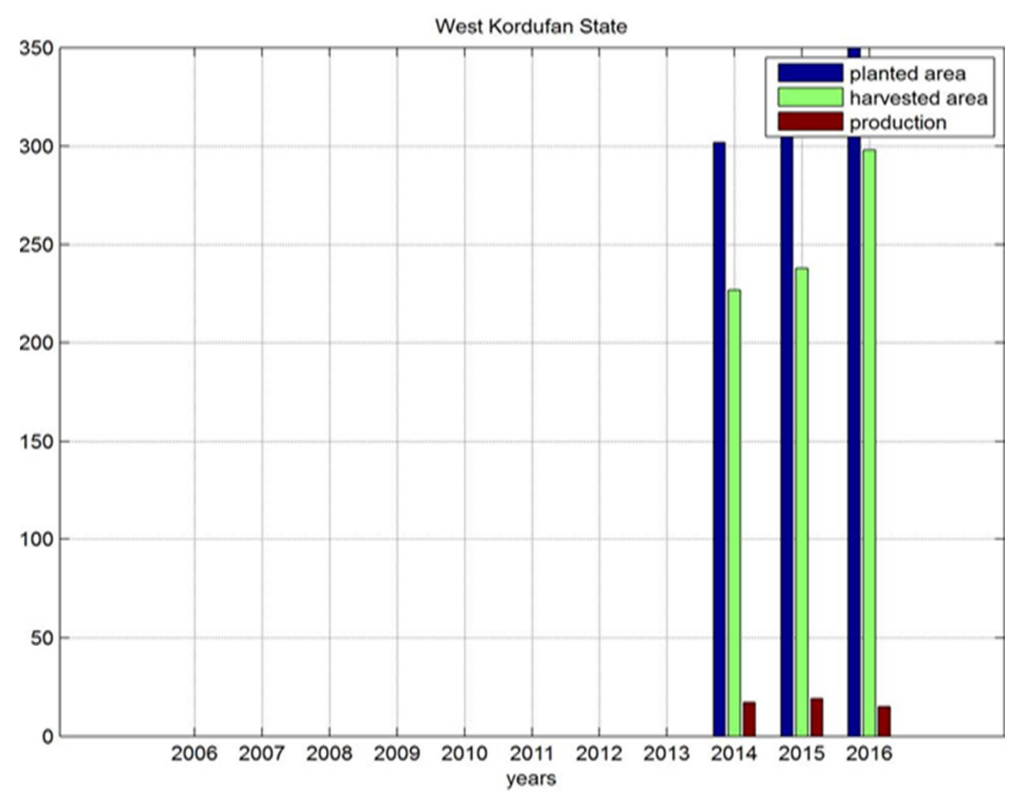

Figure 6. Data Analysis of West Kordufan State.

The production of the year 2015 is the highest production, while the planted area was 305 , and the harvested area 238 because increase of rains, in 2014 - 2016 for the less of production reason for climactic factor.

\subsection{Data Analysis of South Kordufan State}

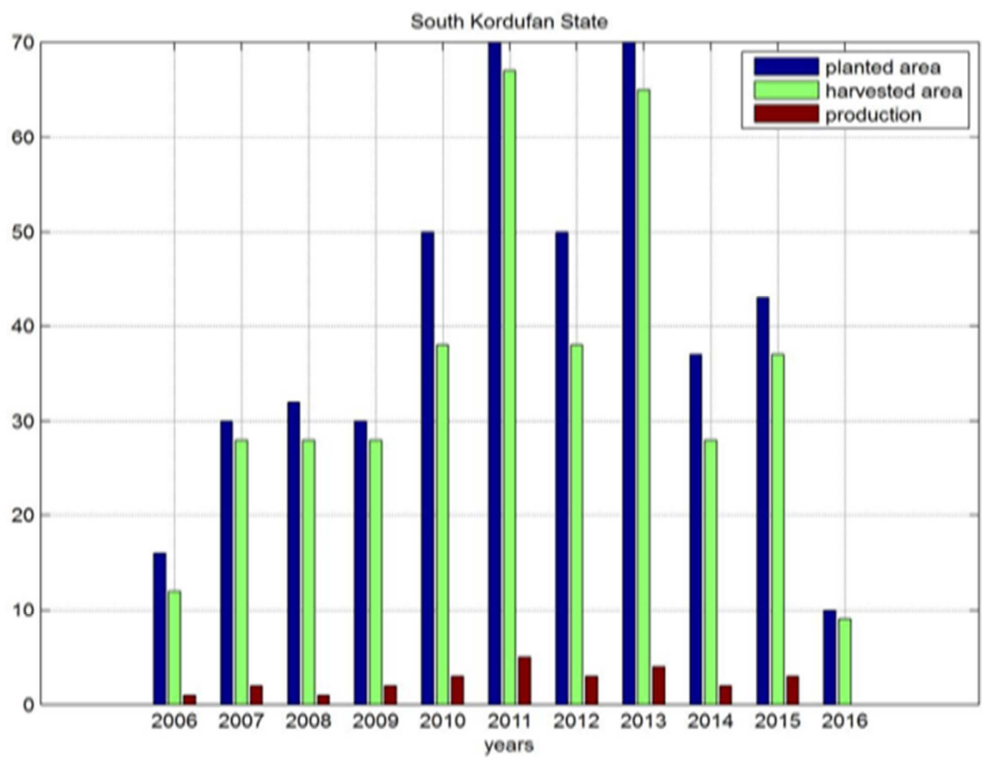

Figure 7. Data Analysis of South Kordufan State.

South Kordufan State is the most equilibrium, while we find that the normal production for the planted and harvested area Reasons:

In Southern Kordufan the soil is muddy and that encourages growing heavy Roselle seeds, abundance of rain which ranges between $400-700 \mathrm{~mm}$, with the possibility of reaching $900 \mathrm{~mm}$, agriculture is mechanized , during 2008$2016 \mathrm{~mm}$ rainfall was relatively scarce compared with the 
previous years and in 2008, 2016 production was not higher because Lack of rain.

\section{Results}

After analysis data, the functions of Matlab program is evaluated and final results are discussed. Results obtained from the functions are compared with the results obtained by each year. The comparison results are shown in Finger 5, 6, 7 for the all states, results show that out of 10 years for planted, harvested area and production. Table 4 shows the functions results which presents all three states. It is noticed that there is the difference between the results of the Matlab functions and the results obtained by designed functions achieve followed results in table 4.

A problem that affects is that parsimonious of rains civil war is neither nonplanted area, harvested area and then not existed production. To solve this problem different reads of different types and stages taken at different times are taken from different areas.

Table 4 shows the three states of hibiscus. These inaccurate results are due to change of direction and reasons.

Table 4. Results of the Matlab Functions.

\begin{tabular}{llll}
\hline The Production & North Kordufan & West Kordufan & South Kordufan \\
\hline High & 2011 & 2015 & 2011 \\
Low & 2014 & 2016 & $2006-2007$ \\
Not Found & - & - & 2016 \\
& 1. Increase of rains & 1. Increase of rains & 1. Increase of rains \\
The reasons & 2. decrease of rains & 2. decrease of rains & 2. decrease of rains \\
& 3. Parsimonious of rains & 3. Parsimonious of rains & 3. Parsimonious of rains \\
\hline
\end{tabular}

\section{Discussion}

The performance of the Matlab functions designed was evaluated in (detection state and counting of hibiscus for planted, harvested area and production) for North, East and South Kordufan states Matlab functions were selected to the test data for validation.

This study has pursued a new style in analysis and calculating of data by using Matlab program other studies that used the manual processing. this study was a great success in the hibiscus production as sample by using high quality program (Matlab) and very accuracy in the results but failed something prediction results in future . and solved this problem by use map of rains as reference contain all levels of rains after this solving achieved more than $95 \%$ accuracy for analysis data.

Found similarities between this study and other studies in the analysis and counting of data in use of computer processing however, this study was characterized by used a lot of data from different area for last 10 years as well as this study was for three states.

\section{Conclusion}

This study presents computers processing systems for hibiscus by using Matlab program for analysis and counting and classification of data. The first is data analysis functions and the second is a counting and classification of data hibiscus into three states (North, East and South Kordufan states). Data gathering for 90 reads from different resources including the online database provided by the ministry of agriculture. All this data passed through code that designed by Matlab program for analysis and counting data in the analysis obtained bar graph for all data for each state. And counting production for select state of hibiscus production (high, low and non-exist).
A new algorithm is designed based on the type of data and compared results with each year in one state, Matlab functions results showed excellent efficiency with 95\% accuracy for analysis, counting and classification.

\section{References}

[1] Abdelatif Ahmed, Bahaeldeen Babiker, Rosell (HibicusSabdariffaL) in Sudan, NCR, Sudan, 2009.

[2] Andrew knight, Basics of Matlab and Beyond, CRC Press LLC, London, 2000.

[3] Brian R. Hunt, Jonathanm. Rosen berg, Ronald L. Lipsman, Aguide to Matlab for Beginners and Experienced users, John. wiley and sons, INC, New york, 2004.

[4] David Halpering, Howard B. Wilson, Louis H. Turcotte, advanced mathematics and Mechanics Application Using Matlab, Third Edition, Chapman \& HALL/ CRC/ London, 2003.

[5] David Houcque, Introduction to Matlab for Engineering Students, North Western University, 2005.

[6] David McMahon, Matlab Demystified, Mc GrowHillg, Chicago, 2007.

[7] Dr. Guma Kundg Komey, Maria Hahnekamp, Hibiscus production and Market chanisin Um Ruwaba and El-Rahad, North kordofan, University Jub, University of Halle, Sudan , Germany, 2010.

[8] Dr. SikanderM. Mirza, Introduction to Matlab, PIEAS, 2003.

[9] JOHNO. ATTIA, Electronics and Circuit Analysis using Matlab, CRC, Boca, Raton, 1999.

[10] Kermit Sinmon, Timothy A. Davis, Matbal Primer, Sixth Edition, ACRC Press Company, Washington, 2002.

[11] Kermit Sinmon, Timothy A. Davis, Matbal Primer, Seventh Edition, ACRC Press Company, New York, 2005. 
[12] Kermit Sinmon, Timothy A. Davis, Matbal Primer, Eighth Edition, ACRC Press Company, London, 2011.

[13] O. ThomasHoLL and Patrick Marchand, Graphics and Guis with Matlab, Third Edition CHAPMAN \& HALL/CRC, New York, 2003.

[14] Stormy Attaway, Matlab A practical Introduction to Programming and Problems Solving, Elsevier, Tokyo, 2009.

[15] The Mathworks, Getting Starting with Matlab, TheMathworks, Ink, 2001.
[16] Wendy L. Martinez, Computation Statistics Hand Book with Matlab, Chapman \& Hall /CRC, Washington, 2002.

[17] Agricultural Research Station while North Kordofan, Second, 2010.

[18] Ministry of Agricultural statistics, 2016-2017.

[19] http://cimss.ssec.wisc.edu/wxwise/class/aos340/spr00/whatis matlab.htm. 